\title{
Prevention and Treatment of Cardiovascular Diseases in Women During the Premenopausal Period
}

\author{
Basieva L.M. ${ }^{1,{ }^{*}}$ Kabulova I.V. ${ }^{2}$ Popova L.S. ${ }^{2}$ Tsallagova L.V. ${ }^{2}$ Maisuradze L.V. ${ }^{2}$ \\ Alborov D.K. ${ }^{3}$ Dzagoeva Z.L. ${ }^{4}$ Gabueva Ya.O. ${ }^{5}$

\begin{abstract}
${ }^{1}$ Department of Internal Medicine 3, North-Ossetian State Medical Academy of the Ministry of Health of the Russian Federation, Vladikavkaz, Russia

${ }^{2}$ Department of pathology of mother and child, Institute of Biomedical Research - a branch of Vladikavkaz Scientific Center of the Russian Academy of Sciences, Vladikavkaz, Russia. Department of Obstetrics and Gynecology 1 of the North Ossetian State Medical Academy of the Ministry of Health of the Russian Federation, Vladikavkaz, Russia

${ }^{3}$ Maternity hospital 2, Vladikavkaz, Russia

${ }^{4}$ Department of obstetrics and gynecology 1 of the North Ossetian State Medical Academy of the Ministry of Health of the Russian Federation, Vladikavkaz, Russia

${ }^{5}$ North Ossetian State Medical Academy of the Ministry of Health of the Russian Federation, Vladikavkaz, Russia,

*Corresponding author. Email: akusherstvo_1@mail.ru
\end{abstract}

\begin{abstract}
The work is based examination and treatment of 65 women of the premenopausal period. Assessment of daily blood pressure was carried out by the daily monitoring of blood pressure (DMBP); the state of central hemodynamics was studied by tetrapolar transthoracic rheography, the cerebral hemodynamics was studied by transcranial dopplerography (TCDH), a comprehensive assessment of the hemostasis system - by standard laboratory methods. "Dry" carbon dioxide baths are an effective method of treatment and rehabilitation of patients with a menopausal syndrome. They improve hemostasis, central and cerebral hemodynamics.
\end{abstract}

Keywords: premenopausal period, 24-hour blood pressure monitoring, tetrapolar transthoracic rheography, transcranial dopplerography, hemocoagulation system, "dry” carbonic baths, hypercapnotherapy

\section{INTRODUCTION}

Due to an increase in life expectancy, health problems of older women are of great importance $[1,2]$. Menopause is a physiological period during which hormonal changes occur. Women with a severe climacteric syndrome (CS) are in a risk group, since violation of the autonomic and hormonal balance is one of the pathogenetic factors in the development of cardiovascular diseases (hypertension and coronary diseases) [3-5]. One of the main methods for the CS prevention and treatment is a hormone replacement therapy. However, given the wide range of contraindications and complications, hormones do not solve this important medical and social problem [6-9]. The so-called "alternative" non-pharmacological methods for the prevention of menopausal disorders are of great interest. They reduce the drug load on the body and improve quality of life. The pathogenetic rationale for the use of dry carbon dioxide baths (SUV) was their positive effect on the cardiovascular system, excitation and inhibition in the central nervous system, the vegetative tone, and oxidative metabolism [10]. The aim of the article is to study the effect of "dry" carbon dioxide baths on the central and cerebral hemodynamics, hemostasis in premenopausal women.

\section{METHODS AND MATERIALS}

The research is based on the survey of 65 women in the premenopausal period. To assess severity of the premenopausal period, the menopausal index (Kupperman index) modified by E.V. Uvarova was used (1983) - The Modified Menopausal Index (MMI). Assessment of the daily schedule of blood pressure was carried out by the method of daily monitoring of blood pressure (DMBP); the state of central hemodynamics was studied by tetrapolar transthoracic rheography, cerebral hemodynamics - by transcranial dopplerography (TCD); a comprehensive assessment of the hemostasis system - according to standard laboratory techniques. The patients were divided into 2 groups comparable in terms of the main clinical and functional characteristics: the main group (35 patients) took dry carbon dioxide baths; the control group (30 patients) received symptomatic traditional therapy, including tranquilizers, antihypertensive drugs, nootropics, antidepressants, vitamin and mineral complexes. The average age of the patients of the main group was $48.1 \pm 0.9$ years, of the control one $-47.6 \pm 1.2$ years. The duration of menopausal disorders ranged from 3 months to 5 years (average $1.7 \pm 1.1$ years). The main complaints were hot flashes to the head and upper part of the body, headaches, 
dizziness, sweating, lability of the pulse and blood pressure, irritability, fatigue, decreased performance, memory loss, conflicts. The premenopausal period was characterized by a high level of extragenital and gynecological morbidity: hypertension $(49.2 \%)$; diseases of the musculoskeletal system (76.9 \%); diseases of the gastrointestinal tract $(53.8 \%)$; varicose $(33.8 \%)$; overweight $(49.2 \%)$; uterine fibroids and cervical diseases (47.7 \%); inflammatory diseases of genital organs (61.5\%). In accordance with the data of the modified menopausal index (MMI), the mild premenopausal period occurred in $10(28.6 \%)$ patients of the main group and in $8(26.7 \%)$ of the control group (the total MMI scores were $29.2 \pm 2,4$ and $28.8 \pm 2.5$, respectively); the average severity was determined in 16 (45.7\%) patients of the main group and in $13(43.3 \%)$ patients of the control group (the total MMI scores were $49.3 \pm 1.8$ and $48.7 \pm 2.1$, respectively); the severe premenopausal period was observed in $11(31.4 \%)$ patients of the main group and in 7 patients $(23.3 \%)$ of the control control group (the total MMI scores were $67.2 \pm 4.6$ and $64.9 \pm 3.8$, respectively)

Static processing was carried out using parametric and nonparametric methods. Statistical calculations were performed in Microsoft Word, Microsoft Excel 2000, and Statistic 6

\section{RESULTS}

After the preliminary examination, patients of the main group took "dry" carbonic baths. A "dry" carbonic bath ("Reabox") is a special semi-automatic chamber in which the body is treated with heated carbon dioxide and the head remains outside. Dosing was carried out according to the following parameters: carbon dioxide concentration $15-20 \%$, temperature $-28-32{ }^{\circ} \mathrm{C}$, duration $15-20$ minutes, 10 procedures.

According to the results of dynamic monitoring, "dry" carbon dioxide baths have a positive effect during the procedure and its aftereffect.

In all 35 women $(100 \%)$ of the main group, the frequency of hot flashes reduced (before treatment, the number of hot flashes was $16.4 \pm 2.3$ per day; after the therapy, it was $3.8 \pm 0.7)$. In the control group, this indicator decreased from $15.9 \pm 3.1$ to $10.2 \pm 1.8$. Under the influence of hypercapnotherapy, the main dynamics was positive and other clinical manifestations (a decreased number of headaches in 28 patients $(80 \%)$, dizziness in 25 patients $(71.4 \%)$, cardialgia in 25 patients $(71.4 \%)$, and heartbeats in 32 patients $(91.4 \%)$; complaints of fatigue, irritability, and emotional lability in 30 patients (85.7\%); sleep improved in 32 (91.4\%) women. Under the influence of the traditional symptomatic therapy, unidirectional favorable changes in clinical symptoms were observed only in $30-45 \%$ of patients.

The data were confirmed by a significant decrease in the modified menopausal index at the end of the hypercapnotherapy: the total MMI score with an average degree of CS severity decreased from $49.3 \pm 1.8$ to $17.6 \pm 1.2$ ( $p<0.05$ ); for severe symptoms, it decreased from $67.2 \pm 4.6$ to $36.8 \pm 2.6(\mathrm{p}<0.05)$. In $19(54.3 \%)$ patients of the main group with mild to moderate CS severity, the clinical manifestations of $\mathrm{CS}$ were eliminated. In the control group, changes in the MMI were significant only in women with a mild degree of CS severity (from $28.8 \pm 2.5$ to $12.8 \pm 1.8$ points; $\mathrm{p}<0.05$ ), clinical manifestations of CS were eliminated in $7(23.3 \%)$ patients.

After the treatment, a decrease in the average daily level of SBP by $14.8 \%(\mathrm{p}<0.01)$ and $5.1 \%(\mathrm{p}<0.05)$, DBP - by $10 \%(\mathrm{p}<0.01)$ and $3.7 \%(\mathrm{p}<0.05)$ was observed. In the main group, the daily average variability of SBP and DBP decreased by $21.1 \%(\mathrm{p}<0.01)$ and $29.8 \%$ ( $p<0.01)$, respectively; in the control group - by $10.6 \%(\mathrm{p}<0.05)$ and $7.9 \%(\mathrm{p}>0.1)$.

In $53.8 \%$ of women with CS, the initial background was characterized by changes in central hemodynamics with a predominance of hypodynamic blood circulation.

When assessing the heart rate in patients of the main group, a decrease in the average heart rate by $10.6 \pm 0.8$ beats per minute $(\mathrm{p}<0.05)$ was observed after 1 month of observation. In patients of the control group, a significant decrease in the heart rate was not observed. The favorable effect of the rehabilitation treatment on the vascular system becomes apparent when comparing the values of the total peripheral resistance: in patients with a hypodynamic blood circulation who received hypercapnotherapy, the HIPS decreased by $33.8 \%$ $(\mathrm{p}<0.01)$, which caused the hypotensive effect. The women improved their myocardial propulsive ability, as evidenced by an increase in SI by $20.4 \%$ (p <0.05), an increase in SI by $20.2 \%$ (p <0.01), and an increase in PV by $20.5 \%(\mathrm{p}<0.01)$. An increase in cardiac inotropism was evident from a decrease in the final diastolic and final systolic volume, which was manifested in a decrease in LV ICR (by $20.6 \%$; p <0.05) and LV ICD (by $21.7 \%$; $\mathrm{p}<0.05$ ). In patients of the control group, an increase in the inotropic reserve of the heart was observed, as evidenced by an increase in UI by $12.1 \%(\mathrm{p}<0.05)$. However, the value of UI was significantly lower than in patients of the main group. As a result of favorable hemodynamic shifts towards, $29(82.9 \%)$ out of 35 patients of the control group changed the initial hypodynamic type of blood circulation to the eukinetic one; in patients of the main group, CHD values became normal.

According to TCD values, in $20(57.1 \%)$ patients of the main group and in $18(60 \%)$ patients of the control group, circulatory deficiency in the cerebral arteries was diagnosed.

When assessing cerebral hemodynamics, we observed a significant increase in linear velocity of the blood flow in the main arteries of the head in $17(85 \%)$ out of 20 patients of the main group and in $5(27.8 \%)$ out of 18 patients of the control group. Hard venous outflow was eliminated: in the main group - in $85 \%$ of the examined patients, in the control group - in $22.2 \%$. Normalization of the resistance index was observed, which indicated a decrease in peripheral resistance in the basin of the arteries. A more convincing decrease in the linear velocity of blood flow and resistance indices $(\mathrm{p}<0.01)$ was 
observed in patients receiving FFM due to regression of deficiency of blood supply to the vessels of both hemispheres, reduced angiospastic reactions and stagnation phenomena.

In the main group, the platelet count increased significantly ( $\mathrm{p}<0.05$ ). Relative blood viscosity decreased from $4.04 \pm 0.02$ to $3.77 \pm 0.01$ conv. $(\mathrm{p}<0.01)$, hematocrit decreased from $48.6 \pm 0.3$ to $38.5 \pm 0.2 \%$ $(\mathrm{p}<0.01)$. From the side of the plasma link of hemocoagulation and fibrinolysis, significant dynamics was observed only in patients of the main group receiving FFS, and was manifested in a decrease in the initially elevated level of the main substrate of blood coagulation fibrinogen from $5.44 \pm 0.31$ to $4.24 \pm 0.24 \mathrm{~g} / \mathrm{l}$ ( $\mathrm{p}<0.05)$. In patients of the control group, there was a tendency to a decrease in the fibrinogen content (from $5.33 \pm 0.25$ to $5.13 \pm 0.24 \mathrm{~g} / \mathrm{l} ; \mathrm{p}>0.05)$. When assessing the cellular link of hemocoagulation, which characterizes the state of the microvasculature, in patients of the main group treated with FFA, a significant decrease in platelet aggregation induced by ADP $(p<0.05)$ and $\operatorname{Adr}(p<0.05)$ was revealed. In patients of the control group, there was a tendency to a decrease in these indicators $\mathrm{p}>0.05$.

\section{CONCLUSION}

Thus, dry carbonic baths have a positive effect on gemodynamics indicators which can reduce the drug load on the body of women during the premenopausal period. The data allow us to consider this method efficient for treating and preventing cardiovascular diseases in women.

\section{REFERENCES}

[1] E.K. Aylamazyan, Gynecology from puberty to menopause, MEDpress-inform, Moscow, 2006, $496 \mathrm{p}$.

[2] V.M. Bogolyubov, Physiotherapy and Balneology, Book 1, BINOM Publ. House, Moscow, 2008, 408 p.

[3] E.M. Vikhlyaeva, Postmenopausal therapy. Effect on menopausal symptoms, course of chronic diseases, and quality of life, MEDpress-inform, Moscow, 2008, 448 p.

[4] V.I. Kulakov, Clinical recommendations, Obstetrics and gynecology, Moscow, 2008, 685 p.

[5] V.P. Smetnik, Menopause medicine, Yaroslavl, 2006, 320 p.

[6] V.I. Podzolkova, Arterial hypertension in women with menopausal syndrome, Clin. Cardiol. Rev. 1 (2005) 12-19.

[7] V.N. Serov, Gynecological Endocrinology, 3rd ed., MEDpress-inform, Moscow, 2008, 528 p.

[8] S.J. Nicholls, K.Wolski, I. Sipahi et al., Rate of progression of coronary atherosclerotic plaque in women, J. Amer. Coll. Cardiol. 49 (2007) 1546-1551.

[9] L.J. Shaw, R. Bugiardini, C.N.B. Merz, Women and ischemic heart disease: evolving knowledge, J. Amer. Coll. Cardiol. 54 (2009) 1561-1575.

[10] I.V. Kabulova, L.V. Tsallagova et al., Modern approaches to rehabilitation therapy for reproductive disorders, Spa Med. 4 (2017) 67-71. 